\title{
Development of a physical map of the soybean pathogen Fusarium virguliforme based on synteny with Fusarium graminearum genomic DNA
}

\author{
Jeffry L Shultz*1, Sikander Ali², Linda Ballard ${ }^{3}$ and David A Lightfoot ${ }^{4}$
}

\author{
Address: ${ }^{1}$ Currently, USDA-ARS, Crop Genetics and Production Research Unit, PO Box 345, Stoneville, MS, 38776, USA, ${ }^{2}$ Institute of Industrial \\ Biotechnology, GC University Lahore, Pakistan, ${ }^{3}$ Mississippi Valley State University, Itta Bena, MS. Currently, USDA-ARS MSA Genomics \\ Laboratory, P.O. Box 345, Stoneville, MS, 38776, USA and ${ }^{4}$ Dept. of Plant, Soil and Agricultural Systems, Genomics and Biotechnology Core \\ Facility, Center for Excellence in Soybean Research, Southern Illinois University, Carbondale IL, 62901, USA \\ Email: Jeffry L Shultz* - jlshultz@hotmail.com; Sikander Ali - alisbiotech@yahoo.com; Linda Ballard - max@tecinfo.com; \\ David A Lightfoot - ga4082@siu.edu \\ * Corresponding author
}

Published: 3 August 2007

BMC Genomics 2007, 8:262 doi:10.1/86/147|-2164-8-262
Received: 9 February 2007

Accepted: 3 August 2007

This article is available from: http://www.biomedcentral.com//47I-2/64/8/262

(c) 2007 Shultz et al; licensee BioMed Central Ltd.

This is an Open Access article distributed under the terms of the Creative Commons Attribution License (http://creativecommons.org/licenses/by/2.0), which permits unrestricted use, distribution, and reproduction in any medium, provided the original work is properly cited.

\begin{abstract}
Background: Reference genome sequences within the major taxa can be used to assist the development of genomic tools for related organisms. A major constraint in the use of these sequenced and annotated genomes is divergent evolution. Divergence of organisms from a common ancestor may have occurred millions of years ago, leading to apparently un-related and un-syntenic genomes when sequence alignment is attempted.

Results: A series of programs were written to prepare $36 \mathrm{Mbp}$ of Fusarium graminearum sequence in 19 scaffolds as a reference genome. Exactly 4,I52 Bacterial artificial chromosome (BAC) end sequences from 2, 178 large-insert Fusarium virguliforme clones were tested against this sequence. A total of 94 maps of $F$. graminearum sequence scaffolds, annotated exonic fragments and associated F. virguliforme sequences resulted.

Conclusion: Developed here was a technique that allowed the comparison of genomes based on small, 15 bp regions of shared identity. The main power of this method lay in its ability to align diverged sequences. This work is unique in that discontinuous sequences were used for the analysis and information not readily apparent, such as match direction, are presented. The 94 maps and JAVA programs are freely available on the Web and by request.
\end{abstract}

\section{Background}

The soybean pathogen Fusarium virguliforme [1-3] (ex Fusarium solani f.sp. glycines) causes soybean sudden death syndrome (SDS). SDS has caused significant yield losses in soybean since it was first noted in 1980 [4]. F. virguliforme is a member of the Martiella section, a Fusarium plant disease complex that contains many related pathogens of the worlds major crops $[5,6]$. Fusarium virguliforme is a hemibiotrophic plant pathogen with a wide host range $[1,5]$. The perfect stage is unknown and all extant strains appear to be of a single mating type [7]. Tools for analysis of this economically important genome have become a high priority, with two BAC libraries and a collection of BAC-end sequences already developed [8-12]. These BAC-end sequences (BES) produce two reads from each discrete clone, and present 1,000-1,600 base pairs of 
genomic DNA for analysis. A map of ordered, joined sequences can result if sufficient BACs are end-sequenced [13].

A reference genome is defined here as a genome to which phenotypic and/or genotypic traits of other genomes may be compared. The comparison of genotypic traits between both related and distant organisms relies on the information available for the reference genome. This information may be as broad as marker order and karyotype and as specific as single nucleotide polymorphisms (SNPs). The decision on which reference genome to use when several are available favors that most closely correlated at both general and specific layers with the organism under study.

Millions of years of divergent evolution may cause problems when comparing distantly related genomes. Even closely related species may share sequence homology, conserved synteny and genome architecture among only a small set of genes and regions after the effects of inversions, replications, translocations, insertions, deletions, duplications and a myriad number of other genetic mutations. Evolutionary pressure can also cause varying genome size and karyotype divergence.

Two genomes were available for use as reference sequence within the Fusarium plant pathogen complex, Giberella zeae (F. graminearum) [14] and Nectria haematococca (F. solani f.sp. pisi) [15]. The cereal crop fungal pathogen Fusarium graminearum had a nearly complete genome sequence [14], a physical map [16] had the same chromosome number and shared many gene expression patterns with the pathogenic F. virguliforme [17] (Yuan and Lightfoot, unpublished).

The objective was to cluster and align discontinuous BACend sequences from $F$. virguliforme in silico, using sequence resources available for $F$. graminearum. Three steps would be taken. First, a method for grouping thousands of discrete DNA fragments from an uncharacterized genome based on the reference genome was developed. Second, the reference genome was annotated for genic (predicted exon) content. Finally, the random BES matches to the reference genome were presented in a linear map format, based on proximity of sequence identities from both ends of each BAC. After these steps, a prediction of regions spanned by the BAC clones could be made and displayed in an informative manner.

\section{Methods}

\section{Sequences used for genome comparison}

In June, 2007, there were 4,152 F. virguliforme BES available on Genbank [8,9]. The BES encoded 2,976,557 bp of Fusarium virguliforme DNA, at an average of 717 bp per record and an overall GC content of 53.9\%. The
36,093,143 bp of Fusarium graminearum reference genome is represented in 511 sequence fragments located within 19 large (>20 Kbp) and 24 small (<20 Kbp) scaffolds that correspond to 4 chromosomes [16] and have a GC content of $48.4 \%$.

\section{Sequence processing}

The creation of an efficient comparison utility required a multi-program process (Figure 1). First, FASTA formatted $F$. graminearum sequence was processed to remove line feeds and headings. Second, the file was scanned for $7 \mathrm{bp}$ sequences that contained all four bases (A, T, G and C). Motifs with all four bases were written to an array composed of 1,020 possible combinations of the first 5 base pairs ( 5 base repeats of $A, T, G$, or $C$ were excluded from the analysis).

The array of sequence motifs and positions was loaded into a second program, followed by the nucleotide-only reference sequence. Fasta formatted BES was input in series, with each BES screened against the F. graminearum genomic sequence. In brief, a five base nucleotide sequence from the BES was used to access the array of sequences, allowing the program to attempt to align only those sequences that shared the first five base pairs of each 15 bp fragment. In addition, the reverse complement of each BES was also tested. Execution times for comparison of 4,152 BES against $36 \mathrm{Mbp}$ of $F$. graminearum genomic DNA were less than three hours. Output from the comparison program was in the form of the reference genome sequence location, BES ID and matching sequence location. If the BES match was from the reverse complement of a sequence a "-" notation was made.

The identification of exonic fragments was carried out using TimeLogic ${ }^{\circledast}$ GeneDetective $^{\mathrm{TM}}$ (Active Motif Inc., Carlsbad, CA) In order to directly relate the exons to the associated BES fragments and to accommodate the processing restrictions of Gene Detective, the identical 36 Mbp single sequence used for sequence comparison was divided into smaller, 999 bp linear order fragments, then tested these against the non-redundant fungal EST database (downloaded from NCBI on Nov 16, 2006; 886,100 fungal ESTs). We used only the top two ranked hits for each sequence and removed overlapping results.

A Windows XP o/s, w/2 GHz processor and $512 \mathrm{MB}$ RAM was used for analyses (other than Gene Detective) performed in this experiment. A map using the 19 large sequence Fusarium graminearum scaffolds as a framework was prepared in MapChart [18], with matching F. virguliforme clones and exon content of $F$. graminearum. Verification of alignments was performed using the BLAST2Sequence utility at NCBI [19]. 


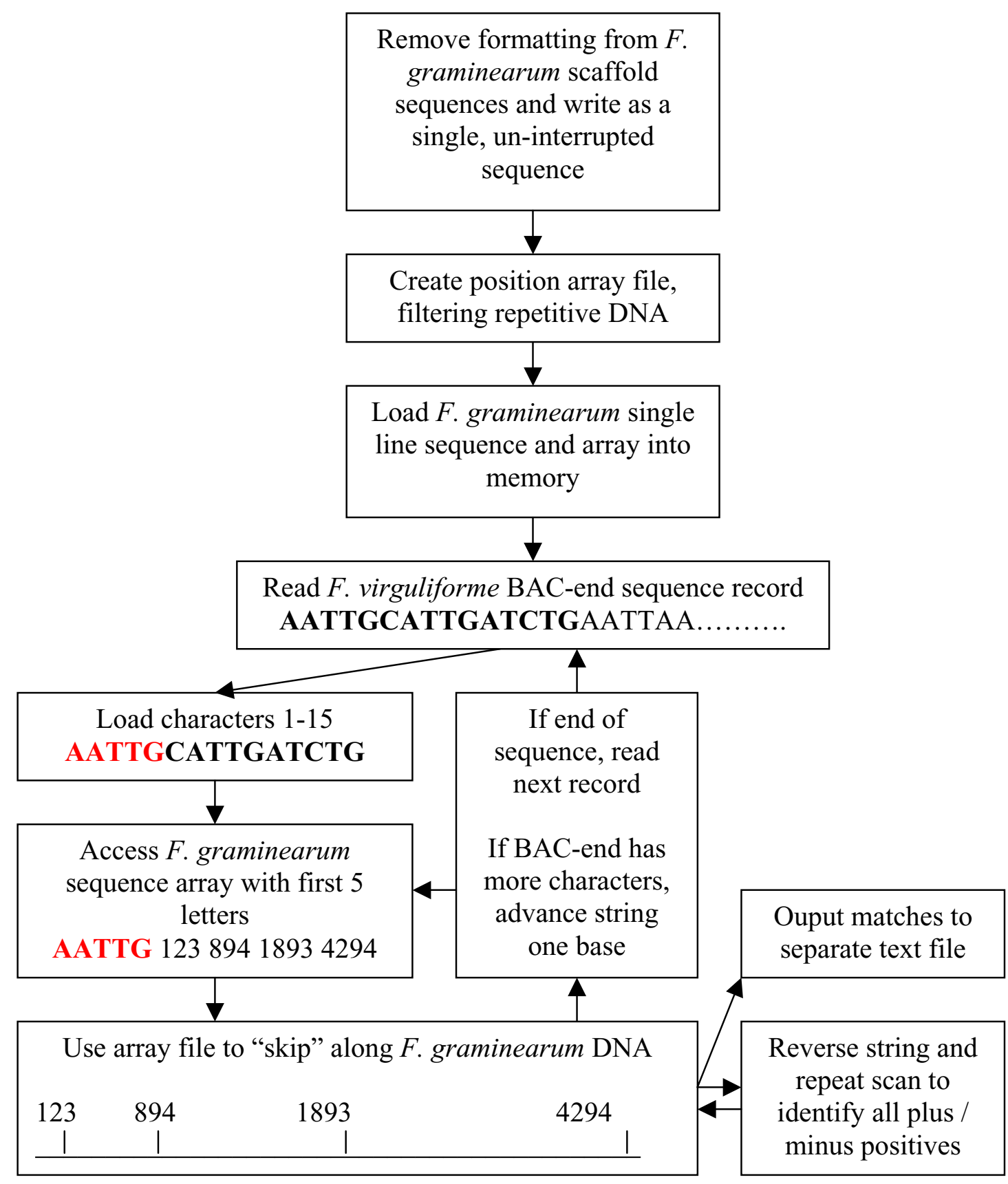

Figure I

Program flow diagram for sequence comparison using $36 \mathrm{Mbp}$ of Fusarium graminearum scaffold sequence as a reference for $4, I 52$ Fusarium virguliforme BAC end sequences. All JAVA code used for this comparison is freely available from the authors. 


\section{Results and discussion}

\section{Fusarium graminearum scaffold-based map}

To expand the use of reference genomes, sequence testing techniques must allow for variations in genetic drift and be capable of reporting associations with varying degrees of certainty [20-22]. In order to meet both of these demands, fifteen base pair "windows" were taken from BAC-end sequences and checked against the reference genome. In addition, each window was reversed and checked against the reference, allowing for change in read direction of BES or of the reference sequence. A fifteen base pair fragment represents a probability of random association every $1 \mathrm{Bbp}\left(4^{15}\right)$. Smaller base pair probes will result in an exponential increase in reported associations. For example, reducing the base pair window to 10 would result in a probability of random association every $1 \mathrm{Mbp}\left(4^{10}\right)$, with loss of accuracy as probability dictates the likelihood of a random match in the reference genome.

As Figure 1 indicates, five bp sequence arrays were used to position the $F$. virguliforme BAC-end probe sequence against the $F$. graminearum scaffold sequence.

These arrays performed two vital tasks. First, they increased processing speed by nearly $1000 \times$ over nonarrayed comparisons. The second advantage related to how the array was prepared. Each 5 bp sequence was written to the array only after the next two bases were added, then this $7 \mathrm{bp}$ fragment was tested for the inclusion of all 4 nucleotides (A, T, G and C). This provided an initial screen of low complexity DNA. It was found that this complexity test was too stringent when tested on 5 bp fragments, and too lax at $8 \mathrm{bp}$.

Identifying conserved regions between $F$. virguliforme BACs and $F$ graminearum DNA led to the detection of a total of 46,590 $15 \mathrm{bp}$ matches. These matches allowed the integration of $F$. virguliforme BAC-end sequence into the linear order scaffold map of $F$ graminearum.

There were two criteria built into this experiment: First, a 15 bp match had to occur; Second, another 15 bp match from the other end of the clone was required within 200 Kbp on the reference sequence. Clones meeting these two criteria were separated into three groups, based on the expected bidirectional nature of BAC end sequence data from the same clone and the unidirectional nature of the F. graminearum scaffolds. The first group were those that had alternating match direction between the two BAC ends and were contained within the same scaffold subsequence. The second group was identical to group 1, except that the two ends span at least two scaffold subsequences. The third group were those that had unidirectional matches between the two BAC ends, regardless of scaffold subsequence position.

A total of 2,560 putatively syntenic associations were identified, encompassing 247,012,452 bp of F. graminearum DNA (6.8 $\times$ genome coverage). A total of 1,339 F. virguliforme clones were identified that exhibited plus and minus strands matching up to 200,000 bp apart. The total DNA covered by +/- alignments was 127,482,241 bp. These clones were separated into 531 that fit within a single scaffold subsequence $(30,915,996$ bp; red clones, Figure 2) and 808 that span more than one subsequence (96,566,245 bp; green clones, Figure 2) In addition, 1,221 clones exhibited plus and plus or minus and minus strands matching up to 200,000 bp apart are indicated on the map in blue. The total DNA covered by $+/+$ or -/matches was $119,530,211 \mathrm{bp}$.

As an example, the match of clone $2 \mathrm{M} 11$ (gi \#s 38262078 and 38262180) with $F$. graminearum scaffold 1.52 is shown in the sequence alignment panel of Figure 2 . The 5 ' match was in two fragments, (181/230 bp, 1e-35; 95/119 bp, $8 e^{-15}$ ) around bp position $3,347,394$ (3.347 on the map). The 3' match was reversed, with 554/683 bp and an expect value of $3 e^{-150}$ around bp position $3,418,000$ (3.418 on the map). Of additional interest was the location of perfectly preserved HindIII restriction sites, 92 bases upstream $(3,347,302 \mathrm{bp})$ in the $5^{\prime} \mathrm{F}$. graminearum sequence and another at the final position $(3,418,308 \mathrm{bp})$ of the 3 ' match (Figure 2, sequence alignment panel). The strength of the sequence matches identified and the nearly perfect correlation with HindIII sites used for cloning the BAC library provide strong evidence of the effectiveness of this sequence correlation technique.

At least 22,614 unique exons encompassing a total of $6,888,863$ bp were detected in the $F$. graminearum scaffold sequences. The match direction of these sequences is indicated with red (forward) or magenta (reverse) text in the map. A total of 94 maps (spanning $\sim 400 \mathrm{~kb}$ each) were generated by this procedure and are available as a selfextracting file [see Additional file 1], which includes a searchable excel file. Each map is viewable as a completely scalable enhanced metafile using the Microsoft ${ }^{\mathrm{TM}}$ Picture and Fax Viewer program.

\section{Future work}

This report is important because it describes the use of $F$. graminearum sequence as a reference to develop genome resources for $F$. virguliforme. The conserved synteny inferred will greatly accelerate the genomic analysis of $F$. virguliforme. The map may also accelerate the discovery of pathogenic gene clusters [16]. Parallels in gene expression underlying the pathogenic mechanisms of both F. virguliforme and F. graminearum may be identified [17]. Strate- 


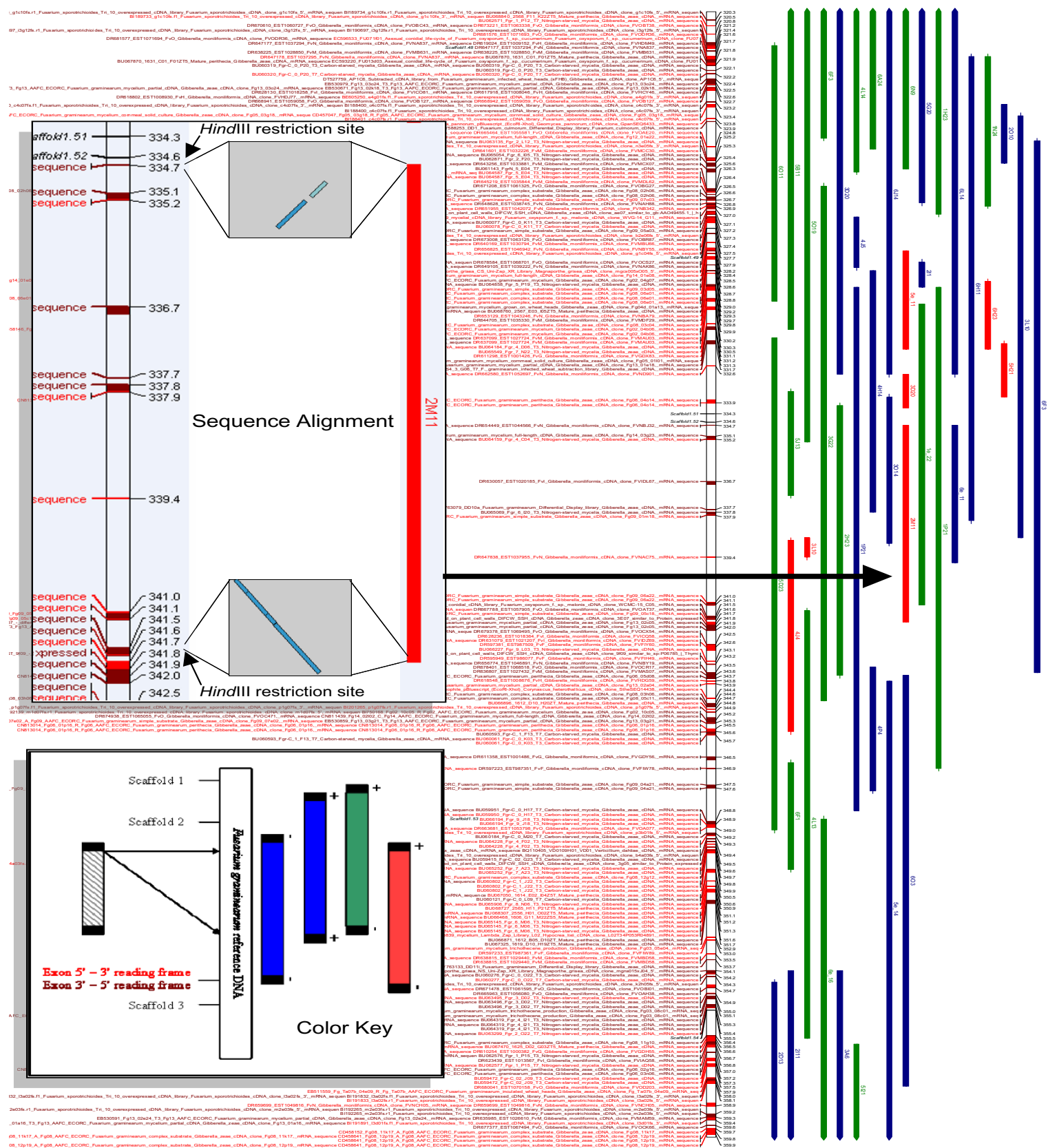

Figure 2

Graphical representation of predicted sequence alignments between Fusarium graminearum reference sequence and Fusarium virguliforme BAC-end sequences. Inset key panel shows how color of predicted match was determined. A match from both ends of the BAC within $200 \mathrm{~kb}$ was required for display. Uni-directional matches are indicated in blue. Reversed order matches are indicated in green if they cross a scaffold marker (indicating a discontinuous sequence) and red if they are entirely within a single, continuous scaffold. Fusarium graminearum exonic fragments are indicated in red ( $\left(5^{\prime}-3^{\prime}\right)$ and magenta $\left(3^{\prime}-5^{\prime}\right)$. Inset sequence alignment panel illustrates the strength of the match between both ends of clone "2MII" and scaffold I.52 (le-35 top and 3e150, bottom) The presence of Hindlll restriction fragments was verified in the F. graminearum sequence, as would be predicted by the position of $2 \mathrm{MII}$. 
gies to reduce yield loss to SDS [4] may also be developed. For example, because F. virguliforme is a member of the Fusarium plant disease complex which contains many related pathogens of all the worlds major crops $[5,6]$, development of genome based fungicides and seed treatments for one may have broad utility. Since F. virguliforme is a hemibiotrophic plant pathogen with a wide host range $[1,5]$, tests against many model plant species to identify new resistance genes are underway. Further, since the perfect stage is not known and all extant strains appear to be of a single mating type [7], these maps represent a significant genetic tool. Finally, the BAC libraries present opportunities for functional genomics, since the vector is a binary T-DNA capable of both plant and fungal transformation mediated by Agrobacterium strains [8,9].

\section{Conclusion}

The main power of this method lay in its ability to align diverged sequences. Because the program uses relationships between 15 bp sequences, the reference genome can exhibit significant change from the probe sequences, yet still effectively group fragments of DNA. This work is unique in that discontinuous sequences were used for the analysis, information not readily apparent such as match direction are presented and the JAVA programs used are freely available. The 94 maps represent an excellent resource for continuing research on Fusarium virguliforme.

\section{Abbreviations}

BAC, bacterial artificial chromosome; BES, bacterial artificial chromosome end sequence; bp, base pair; SDS, soybean sudden death syndrome; SNP, single nucleotide polymorphism.

\section{Authors' contributions}

JS conceived of the study; SA and DL provided critical review, interpretation of results and funding (DL), LB performed geneic annotation of the $F$. graminearum sequences. All authors read and agreed to the final manuscript.

\section{Additional material}

\section{Additional file 1}

A self-extracting file containing 94 maps generated by short sequence comparisons between Fusarium virguliforme and scaffold sequences from Fusarium graminearum. Each map is viewable using the Microsoft $\mathrm{t}^{\mathrm{m}}$ Picture and Fax Viewer program. The extracted files include a searchable Microsoft ${ }^{\mathrm{TM}}$ Excel file.

Click here for file

[http://www.biomedcentral.com/content/supplementary/14712164-8-262-S1.exe]

\section{Acknowledgements}

Thanks are due to Dr Javed Iqbal, Dr. Chris Town, Dr. K Meksem, Hiro Ishihara, H. Koo, J. Yuan, AJ Afzal and Rubina Ahsan for BAC libraries and $B E S$ data. The research was funded in part by grants from United Soybean Board to MJI and DAL. Any opinions and findings were of authors and USB is not responsible for the content. Physical map development was based upon work supported by the National Science Foundation under Grant No. 9872635. Any opinions, findings, and conclusions or recommendations expressed in this material are those of the author(s) and do not necessarily reflect the views of the National Science Foundation. The continued support of SIUC, College of Agriculture and Office of the Vice Chancellor for Research to DAL is appreciated.

\section{References}

I. Roy KW: Fusarium solani on soybean roots: nomenclature of the causal agent of sudden-death syndrome and identity and relevance of F. solani form B. PI Dis 1997, 81:259-266.

2. Aoki T, O'Donnell K, Homma Y, Lattanzi A: Sudden-death syndrome of soybean is caused by two morphologically and phylogenetically distinct species within the Fusarium solani species complex - F. virguliforme in North America and F. tucumaniae in South America. Mycologia 2003, 95(4):660-684.

3. Rupe JC: Frequency and pathogenicity of Fusarium solani recovered from soybeans with sudden death syndrome. Plant Dis 1989, 73:581-584.

4. Wrather JA, Koenning SS, Anderson TR: Effect of diseases on soybean yields in the United States and Ontario (1999-2002). Plant Health Progress 2003, Online:Doi: 10.1094/PHP-2003-3025-0IRV.

5. Gray LE, Achenbach LA, Duff RJ, Lightfoot DA: Pathogenicity of Fusarium solani f. sp. glycines isolates on soybean and green bean plants. J Phytopathology 1999, I 47:28I-284.

6. O'Donnell K: Molecular phylogeny of the Nectria hematococca-Fusarium solani series complex. Mycologia 2000, 92:919-938.

7. Covert SF, Aoki T, O'Donnell K, Starkey D, Holliday A, Geiser DM, Cheung F, Town C, Strom A, Juba J, Scandiani M, Yang XB: Sexual reproduction in the soybean sudden death syndrome pathogen Fusarium tucumaniae. Fungal Genet Biol 2007, 44(8):799-807.

8. Meksem K, Ishihara $\mathrm{H}$, Koo H, Shultz J, Ali S, lqbal J, Lightfoot DA: End Sequencing of BACs from a fingerprint physical map of the causative agent of soybean sudden death syndrome, Fusarium verguliforme (ex Fusarium solani f.sp glycines). Genbank CG8I 2652-CG80850I 2003.

9. Iqbal MJ, Yuan J, Afzal AJ, Ahsan R, Shultz J, Meksem K, Lightfoot DA: End sequence of Fusarium solani $f$. sp. glycines BAC clones. Genbank BH2 I 4549-BH2 I 4545200 I.

10. Engler FW, Hatfield J, Nelson W, Soderlund CA: Locating Sequence on FPC Maps and Selecting a Minimal Tiling Path. Genome Res 2003, I3(9):2152-2163.

II. Marek LF, Mudge J, Darnielle L, Grant D, Hanson N, Paz M, Huihuang Y, Denny R, Larson K, Foster-Hartnett D, Cooper A, Danesh D, Larsen D, Schmidt T, Staggs R, Crow JA, Retzel E, Young ND, Shoemaker RC: Soybean genomic survey: BAC-end sequences near RFLP and SSR markers. Genome 200I, 44(4):572-58I.

12. Shultz J, Meksem K, Shetty J, Town CD, Koo H, Potter J, Wakefield $\mathrm{K}$, Zhang H, Wu C, Lightfoot DA: End sequencing of BACs comprising a provisional tiling path from a fingerprint physical map of soybean (Glycine max) cultivar Forrest. Genbank. CG8I 2653 to CG826 I 26 (I 3,473 sequences). 2003.

13. Budiman MA, Mao L, Wood TC, Wing RA: A deep-coverage tomato BAC library and prospects toward development of an STC framework for genome sequencing. Genome Res 2000, I0(I): I29-136.

14. Guldener U, Mannhaupt G, Munsterkotter M, Haase D, Oesterheld M, Stumpflen V, Mewes HW, Adam G: FGDB: a comprehensive fungal genome resource on the plant pathogen Fusarium graminearum. Nucleic Acids Res 2006, 34(Database issue):D456-8.

15. DOE: Joint Genome Institute . 2007 [http://www.jgi.doe.gov/]. 
16. Guenther JC: The development and differentiation of Gibberella zeae (anamorph Fusarium graminearum) during colonization of wheat. Mycologia 2005, 97:229-237.

17. Gale LR, Bryant JD, Calvo S, Giese H, Katan T, O'Donnell K, Suga H, Taga M, Usgaard TR, Ward TJ, Kistler HC: Chromosome complement of the fungal plant pathogen Fusarium graminearum based on genetic and physical mapping and cytological observations. Genetics 2005, I71(3):985-1001.

18. Voorrips RE: MapChart: software for the graphical presentation of linkage maps and QTLs. J Hered 2002, 93(I):77-78.

19. Tatusova TA, Madden TL: BLAST 2 Sequences, a new tool for comparing protein and nucleotide sequences. FEMS Microbiol Lett 1999, I 74(2):247-250.

20. Soderlund C, Nelson W, Shoemaker A, Paterson A: SyMAP: A system for discovering and viewing syntenic regions of FPC maps. Genome Res 2006, 16(9): II59-1168.

21. Cantor C, Smith C: Genomics: The Science and Technology Behind the Human Genome Project. First edition. New York, John Wiley and Sons, Inc.; 1999:596.

22. Csuros M, Milosavljevic $A$ : Pooled genomic indexing (PGI): Mathematical analysis and experimental design. 2002.

Publish with Bio Med Central and every scientist can read your work free of charge

"BioMed Central will be the most significant development for disseminating the results of biomedical research in our lifetime. "

Sir Paul Nurse, Cancer Research UK

Your research papers will be:

- available free of charge to the entire biomedical community

- peer reviewed and published immediately upon acceptance

- cited in PubMed and archived on PubMed Central

- yours - you keep the copyright

Submit your manuscript here:

http://www.biomedcentral.com/info/publishing_adv.asp 